\title{
COCHRANE
} CORNER

${ }^{\dagger}$ This review is an abridged version of a Cochrane Review previously published in the Cochrane Database of Systematic Reviews, 2014, Issue 9, doi: 10.1002/14651858.CD002018. pub2 (see www.thecochranelibrary. com for information). Cochrane Reviews are regularly updated as new evidence emerges and in response to feedback, and the Cochrane Database of Systematic Reviews should be consulted for the most recent version of the review.

We thank the Cochrane Review Group for their support in publishing these reviews.

\section{Antidepressant treatment for postnatal depression}

Emma Molyneaux, Louise M. Howard, Helen R. McGeown, Amar M. Karia, Kylee Trevillion

\section{Background}

Postnatal depression is a common disorder that can have adverse short- and long-term effects on maternal morbidity, the new infant and the family as a whole. Treatment is often largely by social support and psychological interventions. It is not known whether antidepressants are an effective and safe choice for treatment of this disorder. This review (an update of a 2001 review) was undertaken to evaluate the effectiveness of different antidepressants and to compare their effectiveness with other forms of treatment, placebo or treatment as usual (TAU).

\section{Objectives}

To assess the effectiveness of antidepressant drugs in comparison with any other treatment (psychological, psychosocial or pharmacological), placebo or TAU for postnatal depression.

\section{Search methods}

We searched the Cochrane Depression, Anxiety and Neurosis Group's Specialized Register (CCDANCTR) to 11 July 2014. This register contains reports of relevant randomised controlled trials (RCTs) from the following bibliographic databases: The Cochrane Library (all years), MEDLINE (1950 to date), EMBASE, (1974 to date) and PsycINFO (1967 to date). We also searched international trial registries and contacted pharmaceutical companies and experts in the field.

\section{Selection criteria}

We included RCTs of women with depression with onset up to 6 months postpartum that compared antidepressant treatment (alone or in combination with another treatment) with any other treatment, placebo or TAU.

Data collection and analysis

Two review authors independently extracted data from the trial reports. We requested missing information from investigators wherever possible. We sought data to allow an intention-totreat analysis. Random effects meta-analyses were conducted to pool data where sufficient comparable studies were identified.

Main results

We included six trials with 596 participants in this review. All studies had a randomised controlled parallel group design, with two conducted in the UK, three in the USA and one in Israel. Meta-analyses were performed to pool data on response and remission from studies comparing antidepressants with placebo. No meta-analyses could be conducted for other comparisons because of the small number of trials identified.

Four studies compared selective serotonin reuptake inhibitors (SSRIs) with placebo (two using sertraline, one paroxetine and one fluoxetine; 233 participants in total). In two of these studies both the experimental and placebo groups also received psychological therapy. Pooled risk ratios based on data from three of these studies (146 participants) showed that women randomised to SSRIs had higher rates of response and remission than those randomised to placebo; the fourth study did not report data on response or remission.

One study (254 participants) compared antidepressant treatment with TAU (for the first 4 weeks) followed by listening visits. It found significantly higher rates of improvement in the antidepressant group than the TAU group after the first 4 weeks, but no difference between antidepressants and listening visits at the later follow-up. In addition, one study comparing sertraline with nortriptyline (a tricyclic antidepressant) found no difference in effectiveness (109 participants).

Side-effects were experienced by a substantial proportion of women, but there was no evidence of a meaningful difference in the number of adverse effects between treatment arms in any study. There were very limited data on adverse effects experienced by breastfed infants, with no long-term follow-up. All but one of the studies were assessed as being at high or uncertain risk of attrition bias and selective outcome reporting. In particular, one of the placebo-controlled studies had over $50 \%$ drop-out.

\section{Authors' conclusions}

The evidence base for this review was very limited, with a small number of studies and little information on a number of important outcomes, particularly regarding potential effects on the child. Risk of bias (e.g. from high attrition rates) and low representativeness of participants (e.g. exclusion of women with severe or chronic depression in several trials) also limit the conclusions that can be drawn.

Pooled estimates for response and remission found that SSRIs were significantly more effective than placebo for women with postnatal depression. However, the quality of evidence contributing to this comparison was assessed as very low owing to the small sample size for comparison (146 participants from three studies), the risk of bias in included studies and the inclusion of one study where all participants in both study arms also received psychological therapy. There was insufficient evidence to conclude whether, and for whom, antidepressant or psychological/ psychosocial treatments are more effective, or whether some antidepressants are more effective or better tolerated than others. There is also inadequate evidence on whether the benefits of antidepressants persist beyond 8 weeks or whether they have short- or long-term adverse effects on breastfeeding infants.

Professionals treating women with severe depression in the postnatal period will need to draw on other evidence, including trials in general adult populations and observational studies of antidepressant safety when breastfeeding (although the potential for confounding in non-randomised studies must be considered). More RCTs are needed with larger sample sizes and longer follow-up, including assessment of the impact on the child and safety of breastfeeding. Further larger-scale trials comparing antidepressants with alternative treatment modalities are also required.

Assessed as up to date: 11 July 2014 